\section{9/11-Ersthelfer: Häufig kognitive Defizite}

Eine posttraumatische Belastungsstörung (PTBS) erhöht möglicherweise das Risiko einer kognitiven Beeinträchtigung. Darauf weisen Untersuchungen hin, die nach den New Yorker Terroranschlägen bei Laienhelfern gemacht wurden. Jeder Fünfte von ihnen hatte eine PTBS entwickelt. Einer Stichprobenuntersuchung zufolge war jeder Siebte aus dieser Gruppe von kognitiven Störungen betroffen: Ein 2014/2015 bei 818 Helfern mit PTBS durchgeführter Test hatte bei 104 Patienten eine kognitive Beeinträchtigung und bei 10 eine mögliche Demenz gezeigt. „Das sind schwindelerregende Zahlen in Anbetracht dessen, dass das Durchschnittsalter der Helfer bei 53 Jahren liegt", so die Autoren.

Alzheimer's \& Dementia 2016; 4:67-75

\section{Leseratten leben länger}

Auch wer keine uralten Eltern hat, muss die Hoffnung auf ein langes Leben nicht aufgeben. Zu den empfehlenswerten Maßnahmen gehört einer US-Studie zufolge das Lesen: Senioren, die täglich in Büchern schmökern, leben durchschnittlich 23 Monate länger als Büchermuffel. 3.635 Personen ab 50 Jahren waren nach ihren Lesegewohnheiten befragt und durchschnittlich 9,5 Jahre lang nachbeobachtet worden. Bücherleser hatten gegenüber Nicht-Lesern einen Überlebensvorteil von $20 \%$. Ein dosisabhängiger Effekt auf die Mortalität zeigte sich bereits, wenn die Probanden mindestens 3,5 Stunden pro Woche mit der Lektüre verbrachten.

Soc Sci Med 2016;164:44-8

\section{Abwarten und Tee trinken}

Für eine prospektive Studie wurden 6.508 Menschen ohne kardiovaskuläre Erkrankungen je nach ihrem Kaffee- oder Teekonsum in Gruppen eingeteilt. Vieltrinker genossen mindestens eine Tasse täglich, Wenigtrinker weniger, Abstinenzler gar nichts. Wie sich zeigte, verkalkten die Gefäße der Vieltrinker deutlich weniger, was mit einer signifikant niedrigeren Inzidenz koronarer Erkrankungen korrelierte. Das galt aber nur für die Teetrinker - Kaffeetrinken hatte weder auf die Verkalkung noch auf die kardiovaskulären Ereignisse einen Effekt. Offen bleibt dabei, ob Teetrinker per se gesünder leben.

Am J Med 2016, online 15. September

\title{
Diabetes kann man sich mit Limo antrinken
}

Eine Metaanalyse zeigt, dass der Konsum von Softdrinks mit einem deutlich erhöhten Diabetesrisiko einhergeht. Die größte Gefahr scheint ausgerechnet von zuckerfreien Limonaden auszugehen.

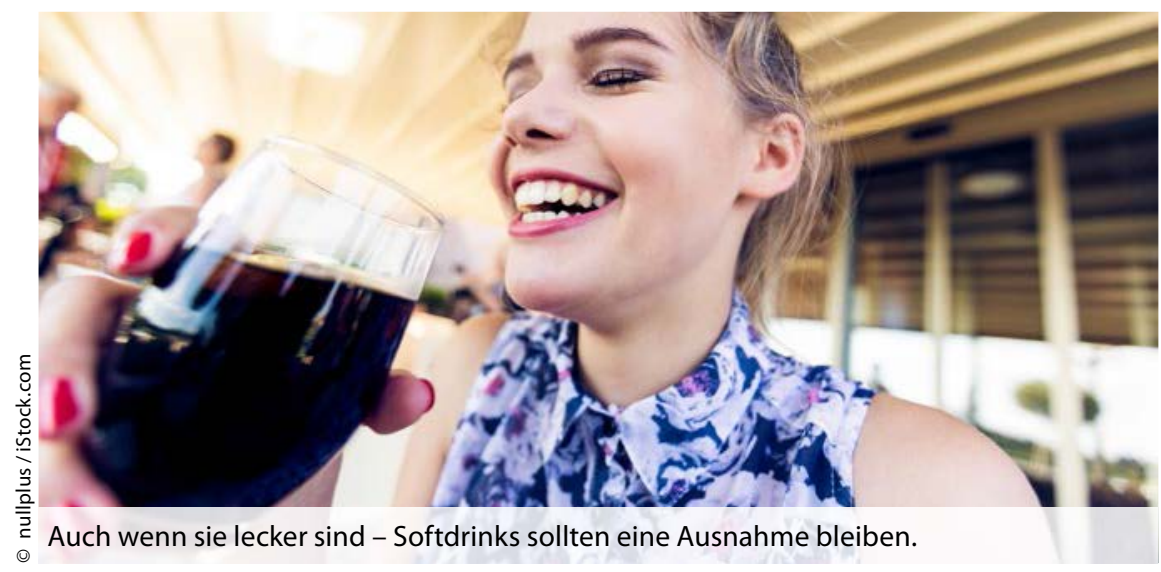

E in systematischer Review mit Meta— analyse sollte den Einfluss süßer Softdrinks und industriell hergestellter Fruchtsäfte auf das Diabetesrisiko erhellen. Dafür wurden alle prospektiven Studien mit nicht diabetischen Erwachsenen bis 2014 eingeschlossen. Endpunkt war jeweils die Verschlechterung der Glukosetoleranz und das Auftreten eines Diabetes. Einbezogen wurden 17 Kohortenstudien mit 38.253 Diabetesfällen und 10.126.754 Personenjahren.

Das Diabetesrisiko stieg um 18\% mit jedem Jahr, in dem täglich ein gesüßter Softdrink getrunken wurde. Für Getränke mit Zuckerersatzstoffen betrug die Risikoerhöhung sogar 25\%, für industriell hergestellte Fruchtsäfte 7\%. Die beiden letzteren Ergebnisse beruhen allerdings auf schwächeren Studien. Ein Vergleich mit nationalen Surveys zur Diabetesprävalenz ergab, dass in den USA von 20,9 Millionen Typ-2-Diabetes-Fällen in zehn Jahren 1,8 Mio. auf erhöhten Softdrinkkonsum zurückzuführen sind. In England sind es bei 2,6 Mio. Fällen nur 79.000. Die Autoren sehen einen klaren Zusammenhang zwischen Softdrinkkonsum und Diabetesinzidenz, der unabhängig vom Übergewicht besteht. Von Zuckerersatzstoffen könnte sogar ein noch größeres Risiko ausgehen.

Imamura $\mathrm{F}, \mathrm{O}^{\prime} \mathrm{C}$ onnor $\mathrm{L}$, Ye $\mathrm{Z}$ et al. Consumption of sugar sweetened beverages, arti_cially swee- tened beverages, and fruit juice and incidence of type 2 diabetes: systematic review, metaanalysis, and estimation of population attributable frac-tion. Br J Sports Med. 2016;50:496-504

\section{KOMMENTAR}

"Ich bekomme Zucker, wenn ich zu viel Zucker esse", hieß es immer von den Patienten. Als Wissenschaftler und Ärzte haben wir versucht, sie zu überzeugen, dass meist eher die Adipositas und das Fett problematisch sind. Aber die Patienten hatten wohl Recht: Ein Softdrink pro Tag über ein Jahr erhöht das Diabetesrisiko um 18\%! Noch erschreckender sind die Daten zu Getränken mit Zuckerersatzstoffen, auch wenn sie noch kein klares Bild ergeben. Das Risikopotenzial könnte aber noch einmal um etwa $30 \%$ höher liegen als bei zuckerhaltigen Softdrinks. Ein Dilemma: Viele Patienten trinken bewusst Light-Getränke, um Zucker zu vermeiden. Womöglich tun sie sich damit nichts Gutes. Was machen wir mit den Daten? Einerseits scheint die Situation in den USA schlimmer zu sein als in Europa, da dort mehr Menschen täglich Softdrinks zu sich nehmen. Es kann aber auch für uns keine Entwarnung sein, da die Trinkhäufigkeit von Softdrinks auch in Deutschland stetig zunimmt. Aufklärung ist daher wichtig. Letztendlich aber wird wohl die Politik eingreifen müssen. Ein Modell, die Nahrungsmittelindustrie für unerwünschte gesundheitliche Nebeneffekte ihrer Produkte haftbar zu machen, könnte sich lohnen.

Prof. Dr. med. P. Schwarz 